\title{
Outage Probability Analysis of Multihop-Multicast Cooperative System
}

\author{
Divya Kumar \\ Department of Electronics and Communication \\ SET, IFTM University, Moradabad
}

\author{
Arun Kumar \\ Department of Electronics and Communication \\ SET, IFTM University, Moradabad
}

\begin{abstract}
In the present paper, a multihop-multicast cooperative wireless network with one source node, $K$ destination nodes, and $M$ relay clusters each consists of $N$ decode-and-forward relay nodes is considered. In each hop of transmission a relay having highest value of received signal-to-noise-ratio (SNR) is selected to forward the source's data. The system is said to be in outage if any one of the destination nodes is in outage, i.e., the received SNR of any destination node is less than a predefined threshold value. The exact value of end-to-end outage probability of the considered system is derived over Rayleigh fading channels. It is shown that with increase in the number of relaying hops the performance of the system improves. Simulations are presented to verify the correctness of obtained analytical results.
\end{abstract}

\section{Keywords}

Cooperative multicast system, Multihop, Outage Probability, Rayleigh fading, Relay selection

\section{INTRODUCTION}

In a latest forecast by Cisco [9] more than eight-fold growth in mobile data traffic is predicted from 2015 to 2020. Correspondingly the spectrum requirement and energy consumption will be raise to connect more devices. To extenuate the instability of fading channels with improved energy and spectral efficiency, among different technologies multiple-input-multiple-output (MIMO) wireless technology is broadly accepted. It has been observed that high capacity and diversity is achieved by employing the MIMO technology [10, 17 20]. Beside this, Multi-hop relaying also emerged as an effective option to realize efficient and trustworthy quality of service (QOS) communication to help wireless cellular network [11, 14 22, 24]. By deploying the one or more relays, a large network split into shorter links, when a direct source- destination link is not possible. Hence with formation of virtual antenna arrays, multihop based cooperative communication system increases the transmission coverage of wireless network by providing spatial diversity [2 7. 13]. In multihop systems, the relay can either decode and forward (DF) [4 7, 13] or amplify and forward (AF) [1 13]. The performance of DF relaying is much better than the AF relaying due to its decoding and forwarding action. [18, 19.22] investigated the performance analysis of DF relaying. In DF relaying process correct or wrong data can be detected and demodulated, depending upon the source relay channels. Therefore DF suffers from the propagation of erroneous relaying, which bounds the use of DF protocol to the multi-hop systems. A maximum likelihood detector can be used for error performance improvement of DF system. Although for relaying source data, the best relay can also be selected [13]. For the diversity and error performance improvement of the DF relaying based multi-hop network system, manifold parallel paths can be used between the source and destination. From these multiple paths, a best multi-hop path can be selected for transmission of the source's data [3]. The best relay selection criterion depends on minimum or harmonic mean of $S \rightarrow R$ and $\mathrm{R} \rightarrow \mathrm{D}$ channel signal-to-noise ratios (SNR). This best relay selection scheme, having the highest SNRs of the minimum of sourceto-relay and relay-to-destination is known the max-min based relay selection [ [5 6 6 12]. For cooperative multi-hop parallel relay network, different path selection techniques, the outage probability and BER analysis investigated over Rayleigh and Nakagami-m fading channels [3 5 5. 6 6].

By providing the multicast and multimedia broad cast, densely populated areas, such as a cricket stadium can be covered easily. However, due to the dynamic changing nature of wireless channels, the multicast transmission of data services is very challenging for the centralized setup [25]. As the number of mobile users (MUs) increases, to confirm all MUs receiving the data, the worst channel condition experienced by all users limited the transmission rate resulting in saturated system throughput [21]. The shadowing, fading and path loss effect in wireless channels can be combat by using relay based cooperative multicast (CM) network [15 16]. The outage probability of DF-based cooperative multicast with the best relay selection (CMRS) over Rayleigh fading channels studied in [15] and for underlay cognitive relay network in [23].

In this paper presents the analysis of the end to end outage probability of DF based multihop cooperative with relay selection (MCMRS) over Rayleigh fading channels. In the proposed system, the best selected relay from the multi-hoped cluster, forward the received signal from the source to another best chosen relay in next cluster. With the propagation of data from different multihop clusters, finally the best relay selected in last hop multicast the signal to a group of destinations (or to multi mobile users, MMUs). For the improvement of the system power distribution with outage probability is calculated.

This paper is organized as follows: Section 2 describes the system model. The outage probability of the considered model analyzed in Section 3 . Numerical results discussed in the Section 4. Finally, conclusion is given in Section 5. 


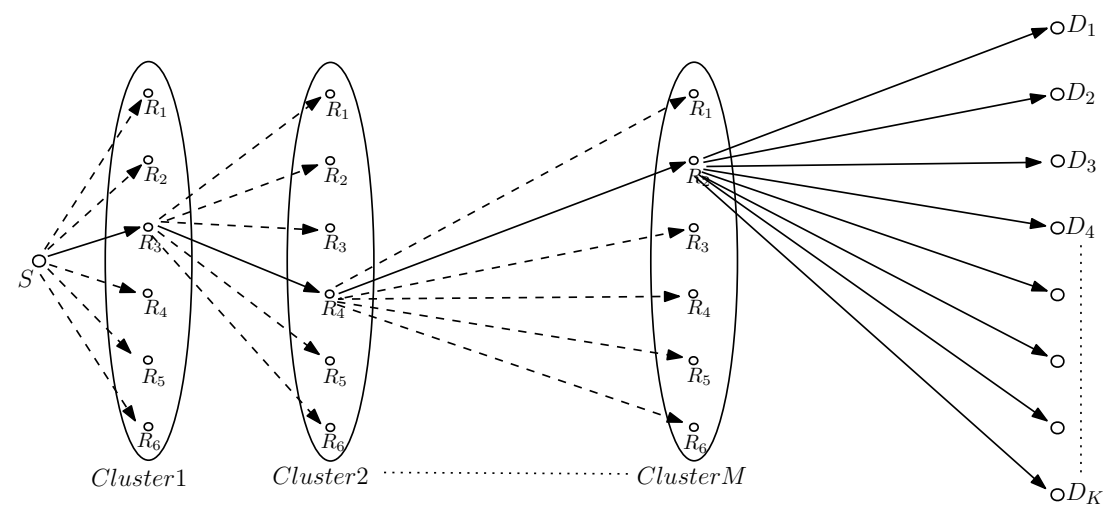

Fig. 1: Multi-hop cooperative multicast system

\section{SYSTEM MODEL}

Our system consists of a source node $S$, multiple destination nodes $\left(D_{i}, i \in 1,2,3, \ldots \ldots, K\right)$, and $M$ relay clusters as shown in Fig. 1 Each relay cluster consists of $N$ number of relay nodes. All the nodes presented in the system are equipped with single antenna that operates in half-duplex mode. It is assumed that no any direct link is available between the source and destination. All the channels presented in the system are assumed to be independent and identically distributed (i.i.d), reciprocal, and flatfading Rayleigh distributed type. The transmit power of each node in the system is denoted by $P_{t}$. Additive white Gaussian noise with $\sigma_{n}^{2}$ variance is presented at each receiving node. The channel coefficient between source and $n$th relay in the first cluster is denoted by $h_{S, n}$, $1 \leq n \leq N$. Let us denote $h_{i, n}$ as the channel from the selected relay in the $i$ th cluster to $n$th relay in the $i+1$-th cluster. The channel coefficient from the selected relay in the $M$ th cluster to the $k$ th destination node is denoted by $h_{M, k}, 1 \leq k \leq K$. The variance of all the channels is assumed to be $\Omega_{h}$. If $X$ is a Rayleigh random variable with variance $\Omega_{h}$, then $Y=X^{2}$ will have exponential distribution. The CDF and PDF of $Y$ are respectively given as

$$
\begin{gathered}
F_{Y}(y)=1-\exp \left(\frac{-y}{\Omega_{y}}\right), \\
f_{Y}(y)=\frac{1}{\Omega_{h}} \exp \left(\frac{-y}{\Omega_{y}}\right) .
\end{gathered}
$$

Protocol: In first time slot source transmits its data towards cluster1. A relay having highest SNR is selected from cluster-1. The selected relay from cluster 1 forwards the data in 2 nd time slot to the next cluster 2 . The same procedure is repeated till the data reaches to all the destinations.

\section{OUTAGE PROBABILITY ANALYSIS}

Let $P_{i}$ denote the outage probability in the $i$ th hop of transmission. The outage probability in one hop is independent of other hops. Therefore the end to end outage probability of the considered system is given by

$$
\begin{aligned}
P_{e 2 e} & =1-(\text { None of the hops are in outage }) \\
& =1-\prod_{i=1}^{M+1}\left(1-P_{i}\right) .
\end{aligned}
$$

The outage probability in first $M$ hops $P_{i}$ for $i=1,2, \ldots, M$, is given by

$$
P_{i}=\operatorname{Pr}\left[\max _{i n=1,2,3 \ldots N}\left\{\gamma_{i, n}\right\} \leq \gamma_{t h}\right],
$$

where

$$
\gamma_{i, n}=\frac{P_{t}\left|h_{i, 1 n}\right|^{2}}{\sigma_{n}^{2}},
$$

and $\gamma_{t h}$ is the required SNR threshold at each node. It is known that all the SNR values are independent of each other. Thus (3) can be rewritten as

$$
\begin{aligned}
P_{i} & =\prod_{n=1}^{N} \operatorname{Pr}\left[\gamma_{i, n} \leq \gamma_{t h}\right] \\
& =\prod_{n=1}^{N} \operatorname{Pr}\left[\frac{P_{t}\left|h_{i, n}\right|^{2}}{\sigma_{n}^{2}} \leq \gamma_{t h}\right] \\
& =\prod_{n=1}^{N} \operatorname{Pr}\left[\left|h_{i, n}\right|^{2} \leq \frac{\gamma_{t h} \sigma_{n}^{2}}{P_{t}}\right] .
\end{aligned}
$$

Now making use of $(1)$ in 3 to get

$$
\begin{aligned}
P_{i} & =\prod_{n=1}^{N}\left[1-\exp \left(\frac{-\gamma_{t h} \sigma_{n}^{2}}{P_{t} \Omega_{h}}\right)\right] \\
& =\left[1-\exp \left(\frac{-\gamma_{t h} \sigma_{n}^{2}}{P_{t} \Omega_{h}}\right)\right]^{N} .
\end{aligned}
$$

In the last hop, if the received SNR at any destination node is less than $\gamma_{t h}$ then outage is declared. Thus the outage probability in the last hop $P_{M+1}$ is given by

$$
\begin{aligned}
P_{M+1} & =\operatorname{Pr}\left[\min _{k=1,2,3 \ldots K}\left\{\gamma_{M, k}\right\} \leq \gamma_{t h}\right] \\
& =1-\operatorname{Pr}\left[\min _{k=1,2,3 \ldots K}\left\{\gamma_{M, k}\right\}>\gamma_{t h}\right] .
\end{aligned}
$$

In (7) all the $K$ SNR values are independent of each other. Therefore (7) can be rewritten as 
Table 1. : Outage probability values of the considered multihop-multicast system for different values of $R$ and $M$ with $\mathrm{SNR}=30 \mathrm{~dB}, N=2, K=4$.

\begin{tabular}{|c|c|c|c|c|}
\hline$P_{e 2 e}$ & $\mathrm{M}=1$ & $\mathrm{M}=2$ & $\mathrm{M}=3$ & $\mathrm{M}=4$ \\
\hline $\mathrm{R}=1$ & 0.0040 & $4.9991 \mathrm{e}-04$ & $1.4814 \mathrm{e}-04$ & $6.2499 \mathrm{e}-05$ \\
\hline $\mathrm{R}=1.5$ & 0.0073 & $9.1390 \mathrm{e}-04$ & $2.7086 \mathrm{e}-04$ & $1.1427 \mathrm{e}-04$ \\
\hline $\mathrm{R}=2$ & 0.0119 & 0.0015 & $4.4438 \mathrm{e}-04$ & $1.8749 \mathrm{e}-04$ \\
\hline $\mathrm{R}=2.5$ & 0.0185 & 0.0023 & $6.8976 \mathrm{e}-04$ & $2.9103 \mathrm{e}-04$ \\
\hline $\mathrm{R}=3$ & 0.0277 & 0.0035 & 0.0010 & $4.3745 \mathrm{e}-04$ \\
\hline $\mathrm{R}=3.5$ & 0.0405 & 0.0051 & 0.0015 & $6.4450 \mathrm{e}-04$ \\
\hline $\mathrm{R}=4$ & 0.0584 & 0.0075 & 0.0022 & $9.3728 \mathrm{e}-04$ \\
\hline
\end{tabular}

$$
\begin{aligned}
P_{M+1} & =1-\prod_{k=1}^{K}\left(\gamma_{M, k}>\gamma_{t h}\right) \\
& =1-\prod_{k=1}^{K}\left[\frac{P_{t}\left|h_{M, k}\right|^{2}}{\sigma_{n}^{2}}>\gamma_{t h}\right] .
\end{aligned}
$$

Using (1) in (8), $P_{M+1}$ can be written as,

$$
\begin{aligned}
P_{M+1} & =1-\prod_{k=1}^{K}\left(\exp \left(\frac{-\gamma_{t h} \sigma_{n}^{2}}{P_{t} \Omega_{h}}\right)\right) \\
& =1-\exp \left(\frac{-\gamma_{t h} \sigma_{n}^{2} K}{P_{t} \Omega_{h}}\right) .
\end{aligned}
$$

Now the end to end outage probability of the considered system is obtained after substituting (6) and (9) in (2) as given below

$$
\begin{aligned}
P_{e 2 e} & = \\
1 & -\left(1-\left[1-\exp \left(\frac{-\gamma_{t h} \sigma_{n}^{2}}{P_{t} \Omega_{h}}\right)\right]^{N}\right)^{M} \exp \left(\frac{-\gamma_{t h} \sigma_{n}^{2} K}{P_{t} \Omega_{h}}\right) .
\end{aligned}
$$

\section{NUMERICAL RESULTS}

In this section, the performance of considered system through MATLAB simulations has been discussed. It is assumed, the source is located at origin in the two-dimensional plane while all the destinations are located at $(0,1)$. The distance between two adjacent clusters is $d=1 /(M+1)$. By considering path loss model the variance of the channels is given by $\Omega_{h}=d^{-\epsilon}$, where $\epsilon$ is called as path loss exponent and the value of $\epsilon$, three is considered.

Fig. 2] studies the variation of outage probability of the considered system versus SNR for $K=6, N=5, R=3 \mathrm{bps} / \mathrm{Hz}$, and $M=1,2,3,4$,and 5 . It can be seen from the figure that the outage probability of the system decreases while increasing the value of $M$. This is due to the fact that, the distance between two nodes will be decreased when the number of hops increases, which further reduces the amount of path loss between the adjacent links. Therefore the performance of system improves with increasing value of $N$.

The variation of outage probability of the considered system versus SNR is plotted in Fig. 3 for $M=3, N=2, R=3 \mathrm{bps} / \mathrm{Hz}$, and $K=1,2,3,4$, and 5 . From this figure it can be observed that the outage probability of the system increases with increasing the number of destination nodes $(\mathrm{K})$. The reason is explained as follows. If the number of destination nodes are increased then the probability that any one node not having the required SNR will increase. Hence the performance of system deteriorates with increase in number of destinations.

Fig. 4 shows the variation of outage probability of the considered system with respect to SNR for $M=3, N=2, K=4$, and $R=1,2,3$, and $4 \mathrm{bps} / \mathrm{Hz}$. It can be noted from this figure that the outage probability of the described system increases with increasing in the value of data rate because of increasing in the value of required SNR threshold $\gamma_{t h}=2^{R}-1$ with increasing the value of $R$.

Table 1 presents the outage probability values of the considered system for different values of $R$ and $M$ with $\mathrm{SNR}=30 \mathrm{~dB}, N=2$, $K=4$. It can be inferred from the figure that the outage probability of the system decreases with increasing the value of $M$ by keeping $R$ value constant. Also, it can be noticed from the table that the outage probability value increases with increasing the value of $R$ when $M$ value is fixed.

\section{CONCLUSIONS}

In this paper studies are made about the performance of multihopmulticast cooperative system with DF relays over Rayleigh fading channels. A simple adhoc relay selection criterion that selects a relay with highest received SNR in each hop is considered for path selection in the proposed system. The exact expression for endto-end outage probability of the considered system is derived. The derived analytical outage probability matches exactly with the simulated outage probability. Uses of multiple relays between source and destinations amend the diversity of wireless network. It is observed that the performance of the system is improved with increasing the number of relaying hops. With increasing either the number of destinations or the required rate the performance was shown to be deteriorated.

\section{REFERENCES}

[1] M.K. Arti, R. K. Mallik, and R. Schober. Beamforming and combining in two-way af mimo relay networks. IEEE Communications Letters, 17(7):1400-1403, 2013.

[2] A. Bansal, M. R. Bhatnagar, A. Hjorungnes, and Z. Han. Low-complexity decoding in df mimo relaying system. IEEE Transactions on Vehicular Technology, 62(3):1123-1137, 2013.

[3] B. Barua, F. Safaei, and M. Abolhasan. On the outage of multihop parallel relay networks. In IEEE 72nd Vehicular Technology Conference Fall (VTC 2010-Fall), pages 1-5, 2010.

[4] M. R. Bhatnagar. Decode-and-forward-based differential modulation for cooperative communication system with unitary and nonunitary constellations. IEEE Transactions on Vehicular Technology, 61(1):152-165, 2012.

[5] M. R. Bhatnagar. Performance analysis of a path selection scheme in multi-hop decode-and-forward protocol. IEEE Communications Letters, 16(12):1980-1983, 2012.

[6] M. R. Bhatnagar. Performance analysis of max-min path selection scheme in multi-hop df cooperative system over nakagami-m channels. In International Conference on Signal Processing and Communications (SPCOM), pages 1-6. IEEE, 2014.

[7] M. R. Bhatnagar and A. Hjørungnes. Ml decoder for decodeand-forward based cooperative communication system. IEEE Transactions on Wireless Communications, 10(12):40804090, 2011. 


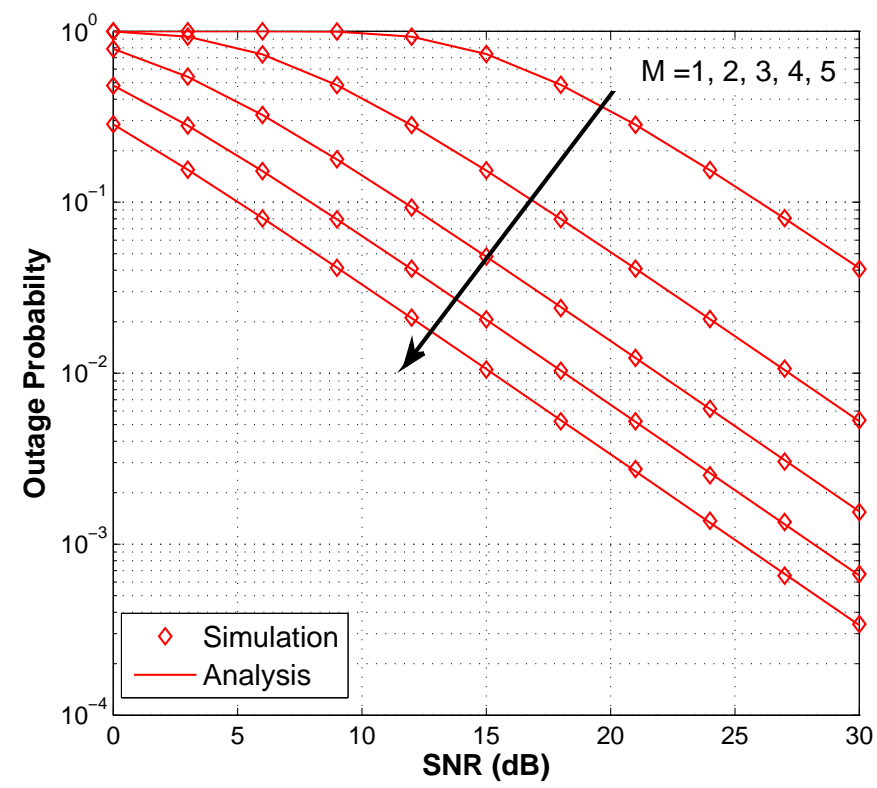

Fig. 2: Outage probability of the considered system versus SNR for $K=6, N=5, R=3 \mathrm{bps} / \mathrm{Hz}$, and $M=1,2,3,4$,and 5.

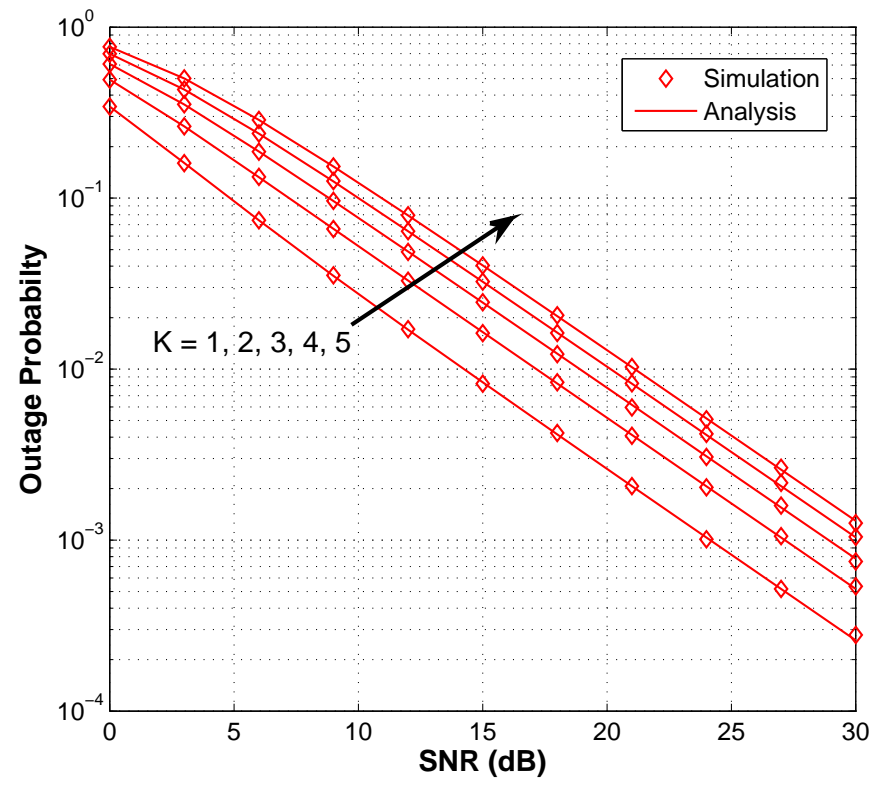

Fig. 3: Outage probability of the considered system versus SNR for $M=3, N=2, R=3 \mathrm{bps} / \mathrm{Hz}$, and $K=1,2,3,4$,and 5 .

[8] S. Butcharoen, C. Pirak, and R. Mathar. On the performance of cooperative multihop communications. In 3rd International Congress on Ultra Modern Telecommunications and Control Systems and Workshops (ICUMT), pages 1-6. IEEE, 2011.

[9] Cisco. Cisco Visual Networking Index: Global Mobile Data
Traffic Forecast Update, 2015-2020 White Paper, 2015. http://www.cisco.com/c/en/us/solutions/collateral/serviceprovider/visual-networking-index-vni/mobile-white-paperc11-520862.html.

[10] A. Goldsmith, S. A. Jafar, N. Jindal, and S. Vishwanath. Capacity limits of mimo channels. IEEE Journal on Selected Ar- 


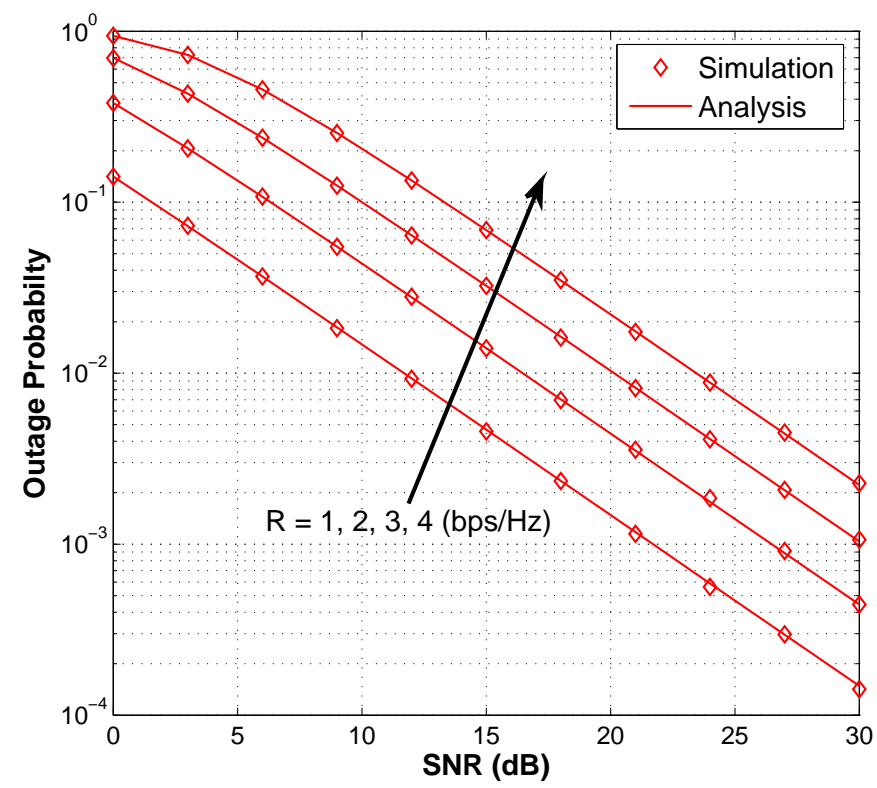

Fig. 4: Outage probability of the considered system versus SNR for $M=3, N=2, K=4$, and $R=1,2,3$, and $4 \mathrm{bps} / \mathrm{Hz}$.

eas in Communications, 21(5):684-702, 2003.

[11] M. O Hasna and M.S. Alouini. Outage probability of multihop transmission over nakagami fading channels. IEEE Cоттиnications Letters, 7(5):216-218, 2003.

[12] I. Krikidis, J. S. Thompson, S. McLaughlin, and N. Goertz. Max-min relay selection for legacy amplify-and-forward systems with interference. IEEE Transactions on Wireless Communications, 8(6):3016-3027, 2009.

[13] J. N. Laneman and G. W. Wornell. Distributed space-timecoded protocols for exploiting cooperative diversity in wireless networks. IEEE Transactions on Information Theory, 49(10):2415-2425, 2003.

[14] L. Le and E. Hossain. Multihop cellular networks: Potential gains, research challenges, and a resource allocation framework. IEEE Communications Magazine, 45(9):66-73, 2007.

[15] I. Ho Lee, H. Lee, and Hyun-Ho Choi. Exact outage probability of relay selection in decode-and-forward based cooperative multicast systems. IEEE Communications Letters, 17(3):483-486, 2013.

[16] C.H. Liu and J. G. Andrews. Multicast outage probability and transmission capacity of multihop wireless networks. IEEE Transactions on Information Theory, 57(7):4344-4358, 2011.

[17] A. F. Molisch and M. Z. Win. Mimo systems with antenna selection. IEEE Microwave Magazine, 5(1):46-56, 2004.

[18] E. Morgado, Inmaculada Mora-Jiménez, Juan J Vinagre, Javier Ramos, and Antonio J Caamaño. End-to-end average ber in multihop wireless networks over fading channels. IEEE Transactions on Wireless Communications, 9(8):2478-2487, 2010.

[19] W. Muenthetrakoon, K. Khutwiang, and C. Kotchasarn. Ser of multi-hop decode and forward cooperative communications under rayleigh fading channel. In Second International
Conference on Intelligent Systems, Modelling and Simulation (ISMS), pages 318-323. IEEE, 2011.

[20] H. Shin and J. H. Lee. Capacity of multiple-antenna fading channels: spatial fading correlation, double scattering, and keyhole. IEEE Transactions on Information Theory, 49(10):2636-2647, 2003.

[21] C. Suh and J. Mo. Resource allocation for multicast services in multicarrier wireless communications. IEEE Transactions on Wireless Communications, 7(1):27-31, 2008.

[22] B. Q. Vo-Nguyen and H. Y. Kong. A simple performance approximation for multi-hop decode-and-forward relaying over rayleigh fading channels. IEICE Transactions on Communications, 92(11):3524-3527, 2009.

[23] L. Yang, J. Chen, Y. Kuo, and H. Zhang. Outage performance of df-based cooperative multicast in spectrum-sharing cognitive relay networks. IEEE Communications Letters, 18(7):1250-1253, 2014.

[24] L. Yang, M. O Hasna, and M.S. Alouini. Average outage duration of multihop communication systems with regenerative relays. IEEE Transactions on Wireless Communications, 4(4): 1366 - 1371, 2005.

[25] H. V. Zhao and W. Su. Cooperative wireless multicast: performance analysis and power/location optimization. IEEE Transactions on Wireless Communications, 9(6):2088-2100, 2010. 